\title{
Ultra Low-Power Digital Demodulators for Short Range Applications
}

\author{
Mehmet R. Yuce ${ }^{1}$ and Ahmet Tekin ${ }^{2}$ \\ ${ }^{1}$ Department of Electrical Engineering, University of Newcastle, NSW, Australia \\ ${ }^{2}$ Dept. of Electrical and Comp. Eng., University of California at Santa Cruz, Santa Cruz, CA 95064-1077, USA
}

\begin{abstract}
In this paper we present extremely flexible and low power digital binary ASK, PSK, and FSK demodulator architectures for short-range applications that use limiter amplifier (i.e. high gain comparator) at front-end. Limiter amplifier can be used at the front-end when the SNR of the received signal is high. This technique converts the analog signal to digital via one bit processing and eliminates mixers, PLL and AGC devices or replaces them with very low power digital counterparts. The rest of circuit is then implemented using digital signal processing (DSP) technique. Detailed system analysis of such demodulators are carried out in this paper. In addition, some new low-size and low-power modulators/demodulators will also be described and analyzed for low-power short-range applications.
\end{abstract}

\section{INTRODUCTION}

There is considerable demand in the development of miniaturized, low power and spectrum efficient wireless communication systems for short-range emerging technologies such as biomedical, remote sensing and Radio Frequency Identification Devices (RFID) applications. For example, current biomedical microelectronics are made wireless so as to increase patient comfort as well as to make robust the implanted device. It is desirable to construct highly integrated and miniaturized communication systems due to the physical limitation of those electronics devices. The ability to develop a low-power device to achieve a reliable communication is critical and important aspect of such small node applications [1][2][3]. Low-power consumption is paramount to enable a long operating life.

To take advantage of low-power and flexibility, it is desirable to implement a demodulator all digitally. Employing a limiter amplifier to convert analog signal to square wave has been an attractive choice so far for RFID and implanted biomedical telemetry systems [3]. This technique is very efficient for low power and size reduction. It eliminates mixers, PLL and AGC devices or replaces them with very low power digital ones. For example, [4] and [5] use ASK (Amplitude Shift Keying), and [6] and [7] use FSK (Frequency Shift Keying) with limiter amplifier at front-end. Achieving lowpower and miniaturized size with a sufficient data rate transmission is among the most important issues in the design of microelectronics systems for such applications.

The purpose of this paper is to address the performance of demodulators that uses limiter amplifier for short-range applications. In addition to reviewing some existing demodulators that have already been published, we will also present alternative PSK and FSK demodulators that can be applied efficiently to short-range applications. These architectures provide high integration and miniature design that consume low power and have small physical size. The system and implementation level details are discussed in detail.

\section{Very Short-Range COMmunications: A System Level APProach}

In short range communications such as RFID technology and implantable electronics, the power consumption and the size of the receiver is more crucial than that of the transmitter. The receivers are mostly passive devices. Thus the receiver power consumption can be traded off with the transmitted power and the transmitter design. In this section we will describe and examine various design parameters for short range communications. Most important parameters that a demodulator is designed for are the bit-error rate (BER) performance, sensitivity, data rate and power consumption. The basic expression for the relationship between the received power and the transmitted power for a wireless communication system is expressed as [10]

$$
\left(P_{R}\right)_{\mathrm{dB}}=\left(P_{T}\right)_{\mathrm{dB}}+\left(G_{T}\right)_{\mathrm{dB}}+\left(G_{R}\right)_{\mathrm{dB}}+\left(L_{s}\right)_{\mathrm{dB}}=\left(P_{T}\right)_{\mathrm{dB}}+\left(L_{T}\right)_{\mathrm{dB}}
$$

where $P_{T}$ is the transmitted power, $G_{T}$ and $G_{R}$ tranmit and receive antenna or coil power efficiency, $L_{S}$ is the free space loss and $L_{\mathrm{T}}$ is the total loss. From the above equation it is clear that the most crucial parameters that define the power level of the received signal are the tranmitter power and the path loss. Designing a low power receiver circuit is directly related to the power level of the received signal. Especially, for short distance communication links, the tight requirements of a receiver circuit are relaxed because of the possibility of obtaining high signal level at the receiver site.

Another common measures of a receiver chracteristic is the sensitivity. The receiver sensitivity can be found by using the equation [9],

$$
S=N_{O}(B) S N R_{\text {Reg }} N F_{T .}=-174_{\mathrm{dBm} / \mathrm{Hz}^{2}}+(B)_{\mathrm{dB}}+\left(S N R_{\text {Reg }}\right)_{\mathrm{dB}}+N F_{T}
$$

Here, $N_{0}$ is the thermal noise, $N F_{T}=\mathrm{SNR}_{\text {in }} / \mathrm{SNR}_{\text {out }}$ is the total noise figure, $S N R_{R e q}$ is the required signal-to-noise ratio (SNR) to get a good performance of $\mathrm{BER}$, and $B$ is the channel bandwidth. The sensitivity of a receiver is defined as the minimum signal level that the system detects with the required SNR. If the signal detected at the front-end is well above the sensitivity level for the worst cases, then a recever design with a high noise figure can be tolerated. Bluetooth receivers are such applications where the minumum signal at the receiver is supposed to be high and so is the noise figure [15]. Such a sytem is easy to design and consumes lower power.

There is legal restriction on transmitter power of short-range devices in many countries [2][3][11]. Regulated transmitter power varies from $10 \mu \mathrm{W}(-20 \mathrm{dBm})$ to $1 \mathrm{~mW}(0 \mathrm{dBm})$. Using 


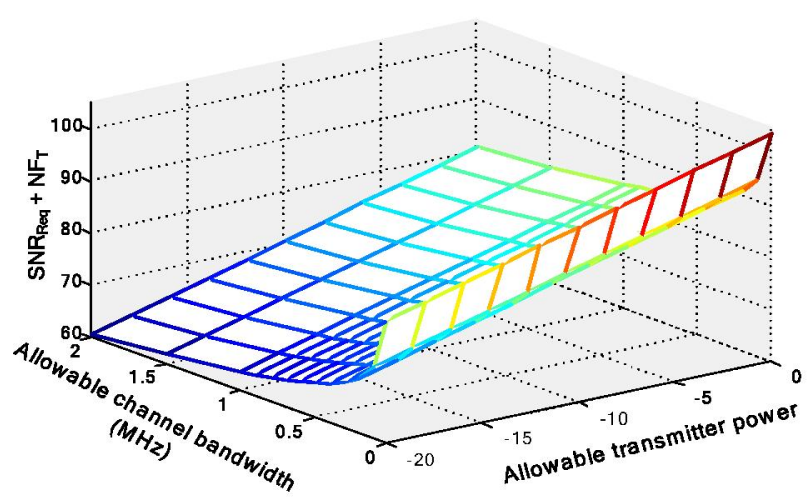

Fig.1 $\mathrm{SNR}_{\mathrm{Req}}+\mathrm{NF}_{\mathrm{T}}$ for short-range communications.

(2), the required SNR plus the noise figure, $\mathrm{NF}_{\mathrm{T}}+S N R_{\text {Req }}=P_{R}+$ $174-B$. Required SNR plus the noise figure values are plotted in Fig. 1 with respect to allowable values of transmitter power and bandwidths for short range applications. Here the total loss is assumed to be $-30 \mathrm{~dB}$, which includes free space loss for a maximum distance of $2 \mathrm{~m}$ as well as antenna losses $^{1}$. As can been seen, the minimum value of $\mathrm{SNR}$ req $+\mathrm{NF}_{\mathrm{T}}$ is higher than $60 \mathrm{~dB}$. An SNR of $30 \mathrm{~dB}$ is quite high and can provide a resonable BER performance lower than $10^{-4}$ (The detail of BER perfomance for the demodulators will be mentioned later.). Even considering interference from nearby channels, this vallue will still be higher than $20 \mathrm{~dB}$ for many cases. A noise figure of $30 \mathrm{~dB}$ is also a relaxed value and allows a very low-power and high gain amplifier design. This conlcudes that a simple limiter amplifier with a high gain can easily be employed at front-end of short-range communication devices that will consume a micro range power [15]. It is thus very attractive from practical view. The reason why most of applications implement power hungry low-noise amplifiers (LNAs) or mixers at front-end is because of the tight requirements of the noise figure which is usually kept below $3 \mathrm{~dB}[9]$.

A block diagram of a receiver with a limiter amplifier can be illustrated as in Fig. 2. A simple bandpass filter (BPF) together with a limiter-amplifier is enough at the front-end. The received signal is passed through a BPF first to reject interference and the out-of-band signals. Then, the limiting amplifier provides enough gain to digitize the input analog signal. However, the operating frequency of limiters is generally limited with Today's technology. It is difficult to design a limiter amplifier operating at higher frequencies since it will require large bandwidth [9][15]. For high frequency applications (usually when $f_{c} \gg 100 \mathrm{MHz}$ ), one down conversion stage is needed as shown in Fig.2-(b). A mixer is used to bring the high carrier frequency to a lower frequency so that the dynamic range of the limiter amplifier can be relaxed.

In RFID and implantable devices, the communication is usually done via inductive link using two coils. As shown in Fig. 3, instead of antennas, a coil pair is used. Adding a tuning capacitor parallel to the coil behaves as a BPF. Most biomedical devices are working with a frequency lower than $100 \mathrm{MHz}$. For this type of applications, L-C tank (BPF), the limiter amplifier and the DSP are the only components in demodulator. In the next section, some low-power implementtation of the DSP part of these devices will be described.

${ }^{1}$ In addition to calculations, a survey of existing device in the literature indicates that the total loss with a resonable antenna design for a maximum $2 \mathrm{~m}$ communication distance is generally lower than $30 \mathrm{~dB}[1]-[6],[11]$.

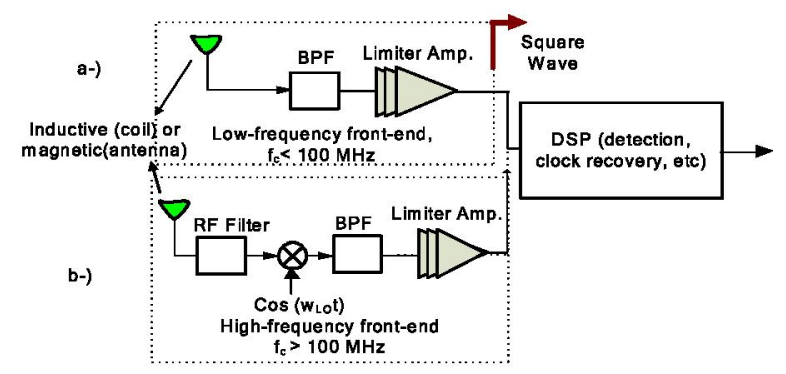

Fig.2. Possible front-end architecture with limiter amplifier.

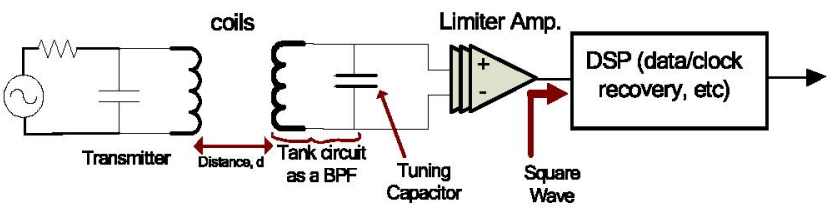

Fig. 3 An inductive link transmission/reception for short-range applications.

\section{Ultra Low-POWER DeMODULATOR ARChITECTURES}

The choice of digital modulation scheme significantly affects the characteristic, performance and physical size of a communication system. The detection of information is done in two ways, coherent or non-coherent. In a coherent scheme, the receiver requires the phase and the frequency information of the transmitted signal. A non-coherent receiver, on the other hand, requires the frequency information only. Coherent demodulators are complex and are usually avoided in low power amplifications. Non-coherent receivers are simple and cheap to build, and thus are widely used in wireless communications [9][10]. There are demodulators that require neither the carrier frequency information nor its phase information. Some of them will be described in this section.

\section{A-) Digital ASK Architecture}

ASK has been commonly used in short-range applications because of its simple modulation and demodulation architecture. $\mathrm{A}$ very simple $\mathrm{ASK}$ demodulation process is described in Fig. 4. After the BPF, the analog ASK signal is compared with a reference signal to eliminate the noise level that may be available during transmission of bit " 0 ". The analog signal is digitized at the output of the comparator (i.e. limiter amplifier). Digital ASK signal is demodulated simply using a frequency divider. For this ASK demodulator, the carrier frequency is an integer order of the bit frequency, $f_{c}=N f_{b}$. The divider should be reset when each time a transition occurs. This can easily be done with a digital logic using the digitized carrier signal.

Although this implementation is very power efficient, it is difficult to arrange the level of the reference signal for high frequencies and high data rates. In addition, ASK performs poor BER performance comparing FSK and PSK. The BER performance of ASK is $3 \mathrm{~dB}$ worse than FSK and $6 \mathrm{~dB}$ worse than PSK [9]. Considering the equation (1) and (2), the transmitter power can be reduced by 2 and 4 using FSK and PSK respectively, instead of ASK signals.

\section{B-) Digital PSK Architectures}

In this section, we discuss low-power, PSK-based receiver architectures using limiter amplifier (i.e. hard-limiter) ${ }^{2}$ via sampling. Fig. 5 shows a low-power implementation of Costas

\footnotetext{
$\overline{{ }^{2} \text { Limiter amplifier is also known as hard-limiter since it is a one bit process[17]. }}$
} 


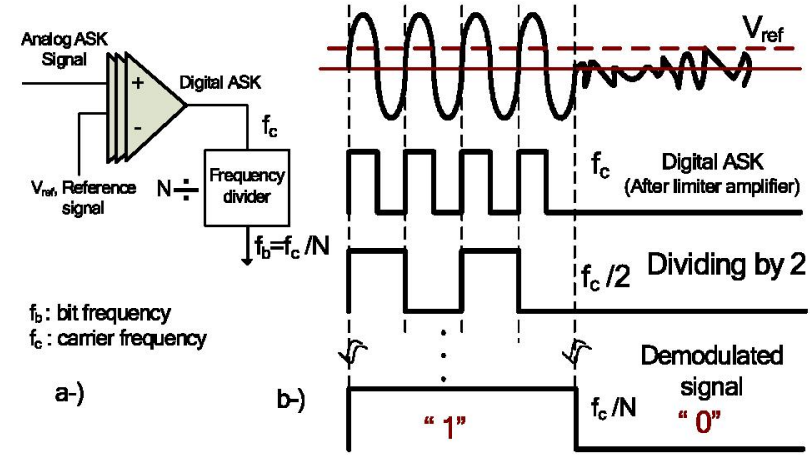

Fig.4. A digital ASK demodulation, (a) schematic of ASK demodulator, (b) timing diagram for ASK demodulation. Here $\mathrm{N}=4$.

loop PSK that employs limiter amplifier at front-end to digitize the analog signal. The digitized signal is then sampled for the required signal processing in the remaining part of demodulation. The numerical controlled oscillator ( $\mathrm{NCO}$ ) generates the reference I/Q signals as square waves. The mixers and low pass filters (LPF's) have been replaced with simple multipliers and accumulators. Mixers can be implemented as simple logic XNOR's, and the low pass filters (i.e. the match filters) are implemented as accumulators or by up/down counters. The accumulators' size depends on the number of samples taken by the decimator.

The received and bandpassed signal can be given by

$$
r_{n}(t)=A \cos \left(w_{c} t+\theta_{n}\right)+n_{n}(t)
$$

where $A$ is the signal amplitude, $w_{c}$ is the radian frequency of the input signal, $\theta_{n}$ is the information phase, and $n(t)$ is the band pass additive white Gaussian with zero mean and singlesided power spectral density equal to $N_{\sigma}$ Assume the sampling frequency is $f_{s}=M f_{\mathrm{c}}$ and after sampled at $t=k T_{s}$, the received signal becomes

$$
r_{n}(\mathrm{k})=A \cos \left(2 \pi k / M+\theta_{n}\right)+n_{n}(\mathrm{k})
$$

After multiplying the received signal with the reference signals using XNOR, the samples at each path are [17]

$$
x_{k}=\left\{\begin{array}{l}
x_{I}(k)=\operatorname{sgn}\left(A \cos \phi+n_{k}\right) \\
x_{Q}(k)=\operatorname{sgn}\left(A \sin \phi+n_{k}\right)
\end{array}\right\}
$$

The phase $\phi$ indicates the phase error between the reference signals and the incoming signal. When the loop recovers the phase precisely, $\phi=0$. The accumulators' outputs are given by

$$
I_{n}(k)=\sum_{k=0}^{K} x_{I}(k) \text { and } Q_{n}(k)=\sum_{k=0}^{K} x_{Q}(k)
$$

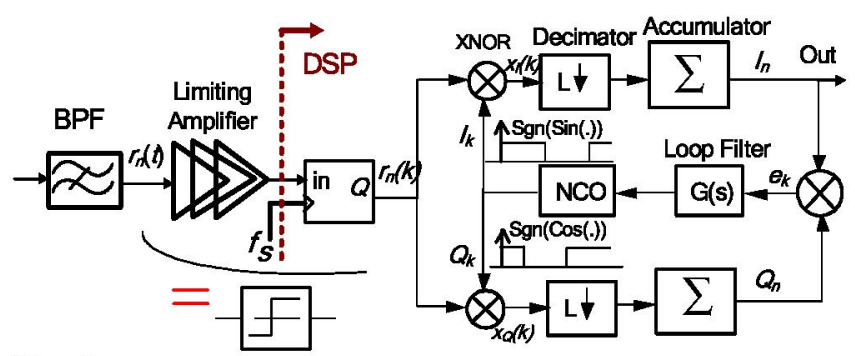

Fig. 5. A low power implementation of digital PSK (Costas loop for BPSK). where $K=f_{\mathrm{s}} T / L$, the number of samples at the accumulators in one symbol duration $T$, and $L$ is the decimator rate. To find the BER of this demodulator, we will use the technique given in [17]. The outputs of the accumulators, $I_{n}$ and $Q_{n}$ are binomial probability mass functions obtained from samples $x_{I}(k)$ and $x_{Q}(k)$. The output signal is taken from the in-phase path. Note that half of the total samples will pass on each path. Thus the probability error of the demodulator $P_{e}=\mathrm{P}\left\{I_{n} \leq 0\right.$ ? $\}$ is

$$
P=\sum_{k=0}^{K / 2}\left(\begin{array}{c}
K / 2 \\
k
\end{array}\right) p^{k}(1-p)^{K / 2-k}
$$

where $p$ is the error probability of each sample in (5) and given by $p=Q(-A \cos \varphi / \sigma)$ and if $B$ is the bandwidth of the BPF, the noise variance is $\sigma_{n}^{2}=N_{0} B$. The BER performance of this digital Costas loop will be plotted later.

Costas loop is a class of phase-locked loops (PLLs) and its stability condition has to be evaluated. Since the Costas loop in Fig. 5 is realized digitally, z-domain technique would be more accurate to analyze its behavior [12]. So far it has been modeled in continuous time domain in literature [13][14]. The discrete model of the Costas loop is depicted in Fig. 6.

The accumulator together with the decimator can be designed with digital counters. Their output is an average of samples. The transfer function, $H_{A D}(z)$, is thus defined as

$$
H_{A D}(z)=\left(1+z^{-1}+z^{-2}+z^{-3} \ldots \ldots .\right) \approx \frac{1}{1-z^{-1}}
$$

A digital loop filter that contains proportional and integral (PI) terms can easily be implemented. The transfer function of the all-digital loop filter is given by [16]

$$
H_{L}(z)=\alpha_{1}+\frac{\alpha_{2}}{1-z^{-1}}
$$

We have two multipliers in the loop. The first one is a simple $\mathrm{XNOR}$ and is represented with a constant $K_{p 1}$. The second one is the phase detector (PD) whose output $\varepsilon$ is given by [13]

$$
\varepsilon=I_{n} \cdot Q_{n} \approx \operatorname{sgn}(\sin (2 \pi \phi))
$$

where $\phi$ is the loop phase errors. Assume this PD is represented with a gain $K_{p 2}$. The closed loop transfer function of all digital Costas loop is given in (11).

$$
H(z)=\frac{\phi_{\text {out }}(z)}{\phi_{i}(z)}=\frac{K\left(\left(\alpha_{1}+\alpha_{2}\right)-\alpha_{1} z^{-1}\right)}{1+K\left(\alpha_{1}+\alpha_{2}\right)-\left(3+K \alpha_{1}\right) z^{-1}+3 z^{-2}-z^{-3}}
$$

where $K=K_{p 1} . K_{p 2}$. $K_{N C O}$ is the total gain in the loop. The stability condition for a discrete-time system is that the poles of the transfer function must be within the unit circuit, $r=|\mathrm{z}|=1$,

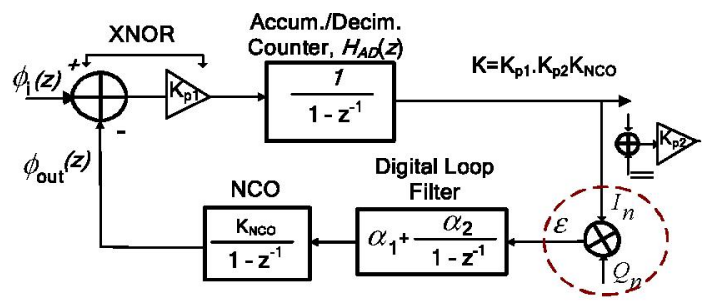

Fig. 6. Discrete time model of all digital Costas loop 
in Z-domain. Stability is mandatory for a closed loop system. Normally when Costas loop is implemented, numerical coefficients, $K, \alpha_{1}$ and $\alpha_{2}$ should be selected such that the poles of (11) are in the unit circle. For the phase acquisition, the counter can be ignored and the loop becomes a second order system. Analysis of a second-order system in the discrete domain is given in [12]. Due to page limitation, further loop analysis of digital Costas loop is omitted in this paper.

Non-coherent scheme of PSK is called differential PSK (DPSK). A digital DPSK using limiter amplifier with sampling is shown in Fig. 7. This demodulator does not need the frequency and the phase information of the carrier signal. The reference signal is taken from adjacent symbols. This reduces the system complexity dramatically since it does not need synchronizations for the carrier frequency and phase. A timing diagram of demodulation process of a hard limited DPSK signal is depicted in Figure 8 . The binary data $a_{k}$ is differentially encoded before transmission. At the receiver site, after the limiter amplifier and the sampling process, the samples of two adjacent symbols are compared within a symbol duration using a simple XNOR gates (i.e. decoder).

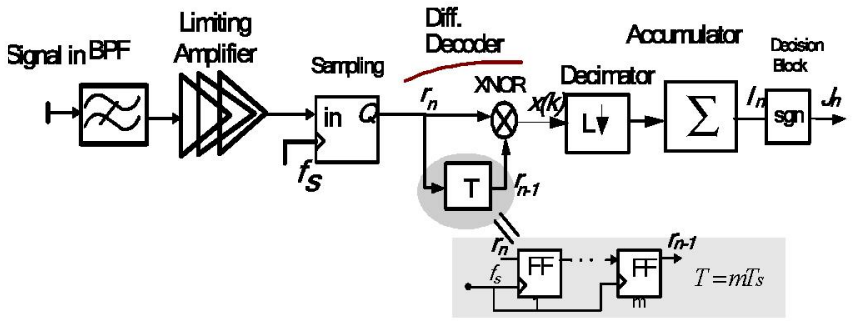

Fig. 7. Digital demodulation of DPSK with implementation detail

The received signal $r_{n}$ and its one symbol delay version $r_{n-1}$ are multiplied using XOR or XNOR gate (depending upon the one used in the transmitter) to obtain $x(k)$. The demodulated signal is given by

$J_{n}=\operatorname{sgn}\left[I_{n}=\sum_{k=0}^{K} x(k)\right]$ and $x(k)=\operatorname{sgn}\left(A^{2} \cos \phi+N\right)$

where $N$ is the total noise at the output of the differential decoder in Fig. 7. It consists of noisexnoise and noisexsignal terms. It is zero mean Gaussian random variable with variance $2 \sigma_{n}^{2}$ for dominant terms [10]. Simulated BER plots of demo-

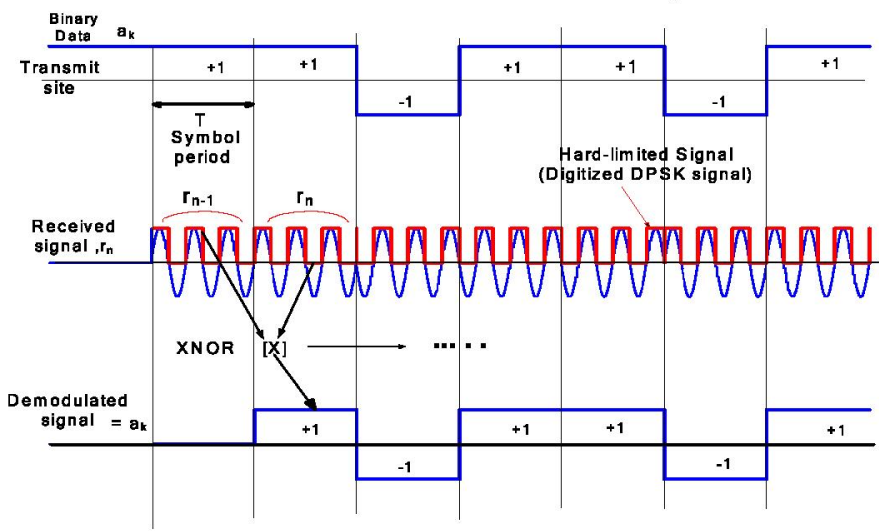

Fig. 8. Timing diagram of digital bit detection of DPSK demodulator.

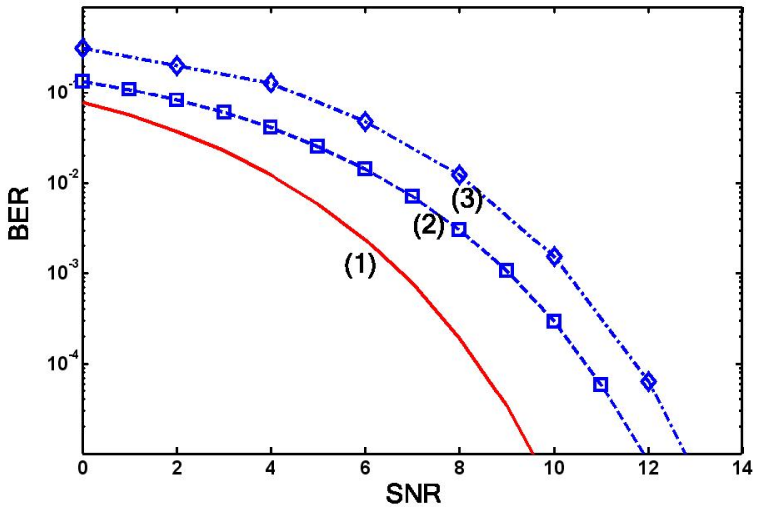

Fig. 9. BER performance of, (1) ideal BPSK,(2) digitized PSK signal in Costas loop(Fig.5),(3) digitized DPSK signal (Fig.7).

dulators in Fig. 5 and 7 are shown in Fig. 9. The BER performance of these demodulators are approximately 2-2.5 $\mathrm{dB}$ lower in terms of SNR comparing to that of an ideal analog demodulation in case of a perfect phase recovery.

For the recevers given in Fig. 5. and Fig. 7 , a sampling frequency, $f_{s}$ is needed in order to sample the input signal. There are many ways to generate the sampling clock at the receiver locally. For low power purpose, the most general way is using ring oscillators [9], as swhown in Fig. 10. However, for high speed aplications, a high sampling frequency might be needed $\left(f_{s}>50 \mathrm{MHz}\right)$. Using a ring oscilator itself would not be an efficient method to generate such a high sampling clock. Thus, for high speed applications, the sampling cock can be generated from a ring oscilator together using an all-digital PLL based clock multiplier, as illustrated in Fig. 10-(b). This sytem is one of the most efficient and simple way for high sampling clock generation in wireless receivers.

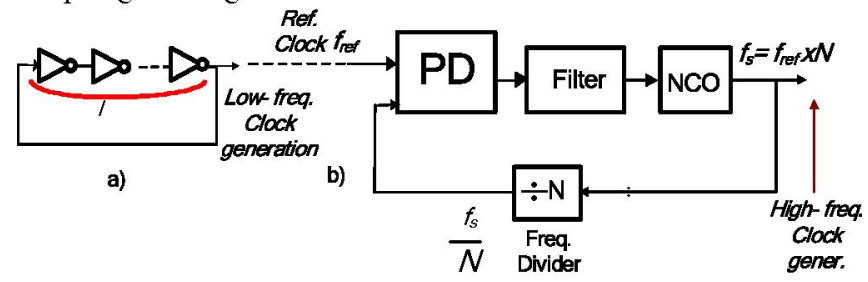

Fig. 10. Ring oscillator and an all-digital frequency multiplier to generate a sampling clock.

\section{C-) Digital FSK Systems}

A very simple method to demodulate FSK signals can be done in a similar way described in Fig.4 [6][8]. In this FSK system, two different frequencies are selected to represent logic " 0 " and " 1 ". For instance, if they are selected as $f_{1}=2 f_{0}$ $=N f_{b}$, two different digital frequency dividers are needed for each carrier cycle.

Another low-power implementation is the classic Vance's FSK demodulator (Fig. 11). This demodulator is designed for electronic pagers and operates at UHF frequency. The limiter amplifiers are used after the carrier is down converted to a lower frequency. In Vance's demodulator, the hard limited I and Q signals are fed into a simple D-type flip-flop to obtain the baseband data. Although very simple in implementation, this scheme exhibits poor BER performance. Here we propose 


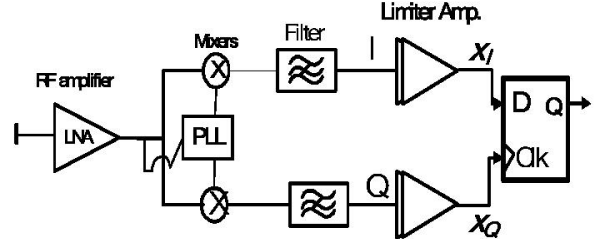

Fig. 11. Vance's FSK demodulator by using a single flip-flop [7]

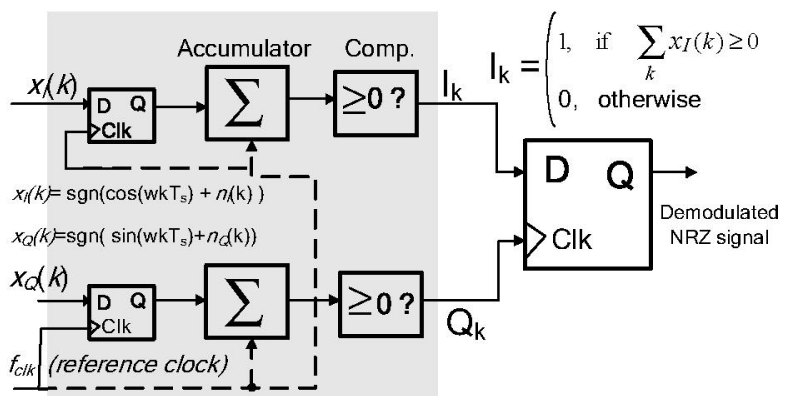

Fig. 12. Enhancing performance of Vance's demodulator. The shadow area is added to eliminate glitches.

a modified version of this demodulator in Fig. 12 [8]. In addition to the famous Vance's demodulator where the signal detection is simply done with a D-type flip-flop [7], we added digital counters/comparators before the detection flip-flop in order to increase the system performance. It takes the in-phase and quadrature signal components $X_{I}$, and $X_{Q}$ as inputs. When limiting a noisy input signal, the signal at the output of the limiter amplifier usually includes glitches. Therefore the demodulator in [7] faces degradation in the signal detection.

The detailed waveforms of the new digital demodulator are depicted in Fig. 13. The sinusoidal quadrature components I and $\mathrm{Q}$ are converted to the digital domain by two limiter amplifiers. These signals are then sampled by a reference clock. The digital comparator compares the total number of samples and decides whether it is less or bigger than zero. Thus, the errors pulses-the glitches in the $\mathrm{X}_{\mathrm{I}}$ and $\mathrm{X}_{\mathrm{Q}}$ are eliminated. The output of the comparator is either a clean " 1 " or " 0 " within pulse duration $\mathrm{T}_{\mathrm{I}}$, as indicated in Fig. 13. The circuits corresponding to both approaches (Fig. 11 and 12) were simulated in Matlab to compare the BER performances. The BER plots are shown in Figure 14. For low SNR values this preprocessing approach outperforms the only D-Flip-flop case.

\section{CONCLUSION}

Some all-digital demodulators have been presented for short range applications. The significance of those demodulators are low-power, extremely low-size and offers the design of lowcost in integrated circuit (IC) technology. Many of these demodulators are made non-coherent and do not require carrier clock recovery due to simplicity. The detailed implementation design issues have been addressed. These receiver architectures can be employed in the applications where the receiver power consumption is more crucial.

\section{REFERENCES}

[1] J. G. Webster, Medical Instrumentation Application and Design. John Wiley \& Sons, Ine. 1998.

[2] FCC Rules and Regulations, Table of Frequency Allocations, Part 2.106, Nov. 2002 (online: http://wireless.fcc.gov/services/).
[3] K. Finkenzeller, RFID Handbook. Wiley \& Sons, Ltd. 2003.

[4] W. Liu, et al., "A neuro-stimulus chip with telemetry unit for retinal prosthetic device," IEEE J. Solid State Circuits, vol. 35, pp. 1487-1497, Oct. 2001.

[5] M. Ghovanloo, K. Beach, K. D. Wise, and K. Najafi, "A BiCMOS wireless interface chip for micromachined stimulating microprobes," in Proc. IEEE EMBS Special Topic Conf. Microtechnology in Medicine and Biology, May 2002, pp. 277-282.2.

[6] M. Ghovanloo and K. Najafi, "A wideband frequency shift keying wireless link for biomedical implants," IEEE Trans. Circuits Syst. II, vol. 51, pp. 2374- 2383, Dec. 2004.

[7] I. A. W. Vance, "An integrated circcuit v.h.f radio receiver," The Radio and Electronic Engineer, vol. 50,no.4,pp. 158-164, April 1980.

[8] A. Tekin, M. R. Yuce and W. Liu, "A low-power FSK modulator/demodulator for an MICS band transceiver," in IEEE Radio and Wireless Symp.(RWS'06), January,2006.

[9] B. Razavi, RF microelectronics. Prentice Hall, 1998.

[10] J. G. Proakis, Digital Communications. $4^{\text {nd }}$ Edition, MeGraw-Hill Book Company. 2001.

[11] Australian Communications Authority, Radio Frequency Planning Group, "Planning for Medical Implant Communications Systems \& Related Devices", Proposals Paper, Oct. 2003.

[12] J. P. Hein and J. Scott, " $z$-domain model for discrete-time PLL's," IEEE Trans. Circuits and Systems, vol. 35, November 1988.

[13] M. K. Simon, "Tracking performance of Costas Loops with Hard-limited in-phase channel," IEEE Trans. Commun., vol. 26, April 1978.

[14] H. K. Philipp et al. " Costas loop experiments for a $10.6 \mathrm{um}$ communications receivers." IEEE Trans. Commun., vol. 31, August 1983.

[15] H. O. Elwan, M. I. Younus, H. A. Alzaher, and M. Ismail, "A bufferbased baseband analog front-end for CMOS Bluetooth receivers," IEEE Trans. Circuit and Systems-II, vol.49, no.8, pp. 545-554, August 2002.

[16] R. E. Best, Phase-Locked Loops: Theory, Design, and Applications. McGraw-Hill Inc., 2003.

[17] P. H. Wu, "The optimal BPSK demodulator with a 1-bit A/D front-end" in Proc. MILCOM'98, vol.3.3, 1998, pages $730-735$.

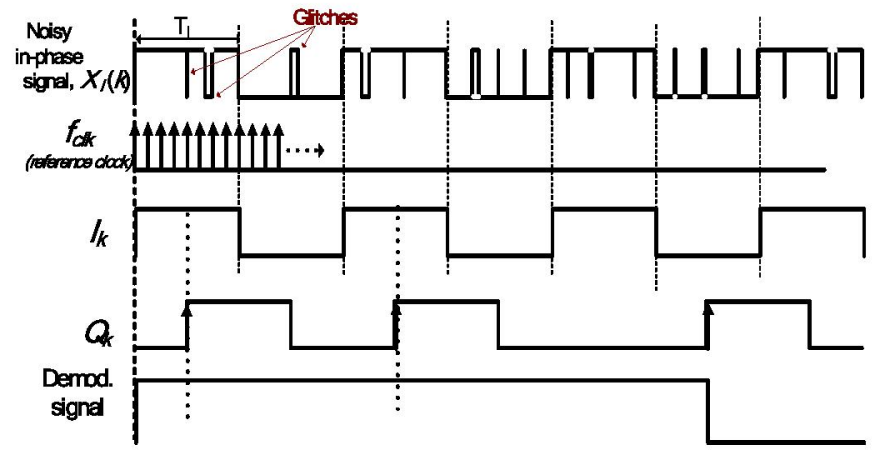

Fig. 13. Signal waveforms of the digital FSK demodulation given in Fig 12

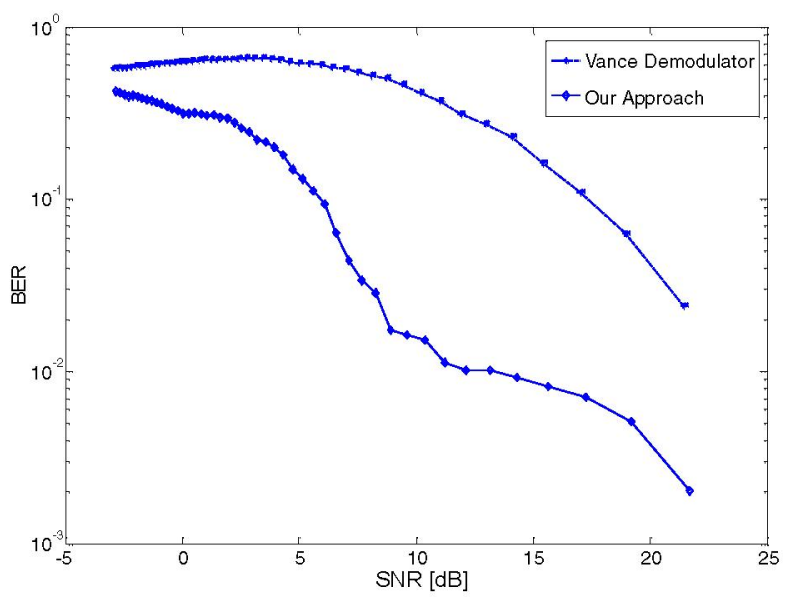

Fig. 14. BER performances of modified Vance's demodulator. 